Letter to the Editor

\title{
A de novo CASK mutation in pontocerebellar hypoplasia type 3 with early myoclonic epilepsy and tetralogy of Fallot
}

\author{
Kazuyuki Nakamura $^{\mathrm{a}}$, Hideo Jinnou ${ }^{\mathrm{b}}$, Kenji Yokochi $^{\mathrm{c}}$, Tohru Okanishi $^{\mathrm{d}}$, Hideo Enoki $^{\mathrm{d}}$, \\ Shigeru Ohki ${ }^{\mathrm{b}}$, Kiyomi Nishiyama ${ }^{\mathrm{a}}$, Hirofumi Kodera ${ }^{\mathrm{a}}$, Mitsuko Nakashima ${ }^{\mathrm{a}}$, Yoshinori \\ Tsurusaki $^{\mathrm{a}}$, Noriko Miyake ${ }^{\mathrm{a}}$, Naomichi Matsumoto $^{\mathrm{a}}$, Hirotomo Saitsu $^{\mathrm{a}, *}$
}

a Department of Human Genetics, Yokohama City University Graduate School of Medicine, Yokohama, Kanagawa, Japan

${ }^{\mathrm{b}}$ Department of Neonatology, Seirei-Hamamatsu General Hospital, Hamamatsu, Shizuoka, Japan

${ }^{c}$ Department of Pediatric Neurology, Seirei-Mikatahara General Hospital, Hamamatsu, Shizuoka, Japan

${ }^{\mathrm{d}}$ Department of Child Neurology, Seirei-Hamamatsu General Hospital, Hamamatsu, Shizuoka, Japan

Corresponding author: Dr. Hirotomo Saitsu

Corresponding author's address: Department of Human Genetics, Yokohama City

University Graduate School of Medicine, 3-9 Fukuura, Kanazawa-ku, Yokohama

236-0004, Japan

Corresponding author's phone and fax: Tel.: +81-45-787-2606, Fax:

$+81-45-786-5219$

Corresponding author's e-mail address: hsaitsu@yokohama-cu.ac.jp

Number of characters in title: 114

Number of words in main text: 302

(C) 2013. This manuscript version is made available under the Elsevier user license http://www.elsevier.com/open-access/userlicense/1.0/ 
To the editor:

In a male patient with pontocerebellar hypoplasia $(\mathrm{PCH})$ type 3 , previously described in this journal [1], we identified a novel hemizygous frameshift mutation in CASK (c.227_228del [p.Glu76Valfs*6]), which occurred de novo. CASK at Xp11.4 encodes a calcium/calmodulin-dependent serine protein kinase belonging to the membrane-associated guanylate kinase protein family. Heterozygous loss-of-function mutations in CASK cause X-linked intellectual disability (ID), microcephaly, and pontocerebellar hypoplasia in female patients [2]. In male patients, hemizygous hypomorphic CASK mutations have been found in ID and FG syndrome; cases had developmental delay, a characteristic face, and complete or partial agenesis of the corpus callosum [3, 4]. On the other hand, hemizygous loss-of-function mutations in $C A S K$ have been reported to cause more severe phenotypes: one case with profound developmental delay, ID, and PCH (c.278 + 1G>A) and two cases showing cerebellar hypoplasia and Ohtahara syndrome (OS) (c.1A $>\mathrm{G}$ or deletion of exon 2) [5, 6].

The present case showed a suppression-burst pattern during the infantile period on electroencephalography and frequent myoclonus from the first day of birth, which were compatible with early myoclonic encephalopathy (EME). OS, which is characterized by early-onset seizures, epileptic spasms, and a suppression-burst pattern on electroencephalography, shares some clinical features with EME. Recently, mutations in STXBP1 have been identified in patients with OS and three patients with EME [7]. Our findings further demonstrate that loss-of-function CASK mutations in males cause early-onset epileptic encephalopathy and $\mathrm{PCH}$, and support the idea that OS and EME may share the same genetic basis.

The patient is also complicated with tetralogy of Fallot, which has never been 
described in association with $C A S K$ mutation. Further studies are required to investigate the causal relationship between loss-of-function mutations in $C A S K$ and congenital heart disease in males.

In summary, a novel frameshift $C A S K$ mutation was identified in a patient with $\mathrm{PCH}, \mathrm{EME}$, and tetralogy of Fallot.

\section{Acknowledgments}

This work was supported by the Ministry of Health, Labour, and Welfare of Japan $(24133701,11103577,11103340,10103235)$; a Grant-in-Aid for Young Scientists from the Japan Society for the Promotion of Science (10013428, 12020465); the Takeda Science Foundation; the Japan Science and Technology Agency; the Strategic Research Program for Brain Sciences (11105137); and a Grant-in-Aid for Scientific Research on Innovative Areas (Transcription Cycle) from the Ministry of Education, Culture, Sports, Science, and Technology of Japan (12024421). 


\section{References}

[1] Jinnou H, Okanishi T, Enoki H, Ohki S. Pontocerebellar hypoplasia type 3 with tetralogy of Fallot. Brain Dev 2012;34:392-5.

[2] Najm J, Horn D, Wimplinger I, Golden JA, Chizhikov VV, Sudi J, et al.

Mutations of CASK cause an X-linked brain malformation phenotype with microcephaly and hypoplasia of the brainstem and cerebellum. Nat Genet 2008;40:1065-7.

[3] Hsueh YP. Calcium/calmodulin-dependent serine protein kinase and mental retardation. Ann Neurol 2009;66:438-43.

[4] Tarpey PS, Smith R, Pleasance E, Whibley A, Edkins S, Hardy C, et al. A systematic, large-scale resequencing screen of X-chromosome coding exons in mental retardation. Nat Genet 2009;41:535-43.

[5] Burglen L, Chantot-Bastaraud S, Garel C, Milh M, Touraine R, Zanni G, et al. Spectrum of pontocerebellar hypoplasia in 13 girls and boys with CASK mutations: confirmation of a recognizable phenotype and first description of a male mosaic patient. Orphanet J Rare Dis 2012;7:18.

[6] Saitsu H, Kato M, Osaka H, Moriyama N, Horita H, Nishiyama K, et al. CASK aberrations in male patients with Ohtahara syndrome and cerebellar hypoplasia. 
1

2

3

4

5

Epilepsia 2012;53:1441-9.

[7] Saitsu H, Kato M, Okada I, Orii KE, Higuchi T, Hoshino H, et al. STXBP1 mutations in early infantile epileptic encephalopathy with suppression-burst pattern. Epilepsia 2010;51:2397-405. 